Ophthalmologe 2011 · 108:709

DOI 10.1007/s00347-011-2365-2

Online publiziert: 6 . August 2011

(c) Springer-Verlag 2011

\author{
S. Kohnen \\ Augen Centrum Dreiländereck, Aachen
}

\section{Rekonstruktive Irischirurgie}

\section{Implantate und Techniken}

Die rekonstruktive Irischirurgie gehört für den Ophthalmochirurgen zu den anspruchvollsten Herausforderungen am Vorderabschnitt des Auges. Erforderlich wird sie, wenn entweder eine funktionelle oder eine kosmetische Indikation vorliegt. Funktionell entstehen bei Irisdefekten oder kompletter Aniridie Blendphänomene, die zu einer erheblichen Beeinträchtigung des Patienten führen können. $\mathrm{Da}$ es sich häufig um traumatische Ätiologien bei jungen Patienten handelt, resultieren hieraus auch folgenschwere Beeinträchtigungen im Straßenverkehr und Berufsleben. Nicht minder beeinträchtigend wirken sich kosmetische Indikationen für Patienten aus. Selbst kleinere Defekte der Iris, die jedem Gesprächspartner direkt ins Auge fallen, können zu einer dauerhaften Irritation und psychischen Belastung führen.

\section{() Indikationen zur rekonstruktiven Irischirurgie können in gleicher Weise funktionell wie auch kosmetisch sein}

Die Ätiologie der Irisdefekte ist vielschichtig. Angeborene Kolobome, komplette Aniridie, Albinismus sind ebenso $\mathrm{zu}$ nennen wie die traumatische $\mathrm{My}$ driasis oder Iristeildefekte nach penetrierenden oder perforierenden Verletzungen. Bei Letzteren finden sich Irisdefekte häufig neben traumatischer Katarakt oder Aphakie. Der Vorderabschnittschirurg wird immer das gesamte Segment betrachten, um eine umfassende Lösung zur Rekonstruktion zu finden. Diese muss operativ nicht zwingend einzeitig erfolgen, auch ein zweizeitiges Vorgehen kann in begründeten Fällen sinnvoll sein.

Die operativen Optionen der rekonstruktiven Irischirurgie werden in diesem Heft dargestellt. Brauweiler erläutert zunächst die klassischen Irisnahttechniken, die immer dann zur Anwendung kommen, wenn noch ausreichend Gewebe zur Verfügung steht. Dauerhaftigkeit der Fadenmaterialien und die atraumatischen Nahttechniken des gleitenden Knotens stehen im Vordergrund. Wenn bei intraoperativen Dehnungsversuchen der Iris erkannt werden muss, dass der Gewebsdefekt nicht überbrückt werden kann, muss auf Irisprothetiken ausgewichen werden. Sektorförmige Lamellen, lose oder an Kapselspannringen fixiert, können kleinere, aber auch größere Defekte oder eine Aniridie abdecken. Die Vorteile und Techniken dieser nichtflexiblen Implantate stellt Wolff ausführlich dar. Alle diese Implantate kommen in der Kleinschnittchirurgie zum Einsatz und bieten Vorteile gegenüber großen Aniridie-Kunstlinsen. Allerdings sind Obige nur für den intakten Kapselsack bei pseudophakem Auge geeignet. Peripher des Linsenäquators können weiterhin Blendphänomene persistieren.

Rekonstruktionen bis in den Sulcus ciliaris ermöglicht erst die künstliche Iris nach Koch, die von Szurman besprochen wird.

Diese flexible Irisprothese mit einem Stützgewebe für genähte Segmentrekonstruktionen bzw. ohne Stützgewebe für die vollständige Aniridie kann individuell für jede Irisfarbe und -zeichnung ange- fertigt werden. Mit ihr lässt sich nicht nur in funktioneller, sondern insbesondere in kosmetischer Hinsicht das Spektrum der Irischirurgie perfektionieren.

\section{( $)$ Periphere Irisdefekte jenseits des Kapselsacks lassen sich mit der künstlichen Iris abdecken}

Das Portfolio der rekonstruktiven Irischirurgie ist auch für den erfahrenen Ophthalmochirurgen anspruchsvoll und weit gefächert. Einen Überblick zu den Implantaten und Techniken finden wir in dieser Ausgabe von Der Ophthalmologe.

Ihr

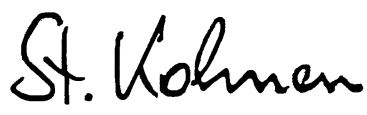

Stephan Kohnen

\section{Korrespondenzadresse}

Dr. S. Kohnen

Augen Centrum Dreiländereck Brüsseler Ring 5a, 52074 Aachen kohnen@augen-acd.de 